\title{
El análisis urbano como una estrategia para la identificación de trayectos y conjuntos con valor patrimonial en la ciudad de San José, Costa Rica
}

\section{Urban analysis as a strategy for the identification of routes and groups with heritage value in the city of San José, Costa Rica}

Rosa Elena Malavassi-Aguilar ${ }^{1}$

Malavassi-Aguilar, R. El análisis urbano como una estrategia para la identificación de trayectos y conjuntos con valor patrimonial en la ciudad de San José, Costa Rica. Tecnología en Marcha. Edición especial. Escuela de Arquitectura y Urbanismo, Diciembre 2020. Pág 154-164.

doi) https://doi.org/10.18845/tm.v33i8.5517 


\section{Palabras clave}

Patrimonio cultural; Valor cultural; Arquitectura; Espacio urbano; Identidad cultural; Cultura; Centro Histórico.

\section{Resumen}

Esta investigación tiene por objetivo desarrollar una estrategia para el conocimiento del patrimonio urbano y arquitectónico en la ciudad de San José, Costa Rica. Con la aplicación del análisis urbano, que toma como referencia las propuestas de los arquitectos italianos Gianfranco Caniggia y Gian Luigi Maffei, se identifican trayectos y conjuntos con valor patrimonial que incluyen aspectos históricos y los imaginarios ciudadanos. La motivación para el desarrollo de esta propuesta es la búsqueda de una figura de protección alternativa al Centro Histórico, de forma que se logre la conservación del patrimonio desde un enfoque integral.

Las fuentes primarias para el desarrollo de este análisis son los mapas y planos que se encuentran en el Archivo Nacional de Costa Rica, que comprenden el período 1841-1982, y las fotografías aéreas de la ciudad de San José del periodo 1945-1998 facilitadas por el Instituto Geográfico Nacional. Con los insumos se construye una cartografía con el uso de un sistema de información geográfica.

Este trabajo se ubica en la línea de análisis del urbanismo y la arquitectura desarrollada por autores latinoamericanos como Marina Waisman, en los que se propone el desarrollo de herramientas propias para el estudio de los bienes patrimoniales locales, de forma que se comprendan las características del contexto. El desarrollo de una herramienta como la propuesta sería de utilidad para los entes encargados de la protección del patrimonio y para las municipalidades en su tarea de gestionar los planes reguladores, en los que deben incluirse las zonas de protección.

\section{Keywords}

Cultural Heritage; Cultural Value; Architecture; Urban Space; Cultural Identity; Culture; Historical Center.

\section{Abstract}

This research aims to develop a strategy for understanding urban and architectural heritage in the city of San José, Costa Rica. With the application of urban analysis, which takes as a reference the proposals of the Italian architects Gianfranco Caniggia and Gian Luigi Maffei, are identified routes and groups with heritage value that include historical aspects and the imaginary of the citizens. The motivation for the development of this proposal is the search for a figure of alternative protection to the Historic Center, so that heritage conservation is achieved from a comprehensive approach.

The primary sources for the development of this analysis are the maps and plans found in the National Archive of Costa Rica, which cover the period 1841-1982, and the aerial photographs of the city of San José from the period 1945-1998 provided by the National Geographic Institute. With these documents, is constructed a cartography with the use of a geographic information system.

This work is located in the line of analysis of urban planning and architecture developed by Latin American authors such as Marina Waisman, in which is proposed the development of own tools for the study of local heritage, so that the characteristics of the context are understood. The 
development of a tool such as the one proposed would be useful for the entities in charge of the protection of heritage and for the municipalities in their task of managing the regulatory plans, in which the protection zones must be included.

\section{Introducción}

En la normativa relacionada con la protección de conjuntos con valor patrimonial, el concepto más utilizado es el de centro histórico. Así sucede en la legislación nacional, la Ley 7555 sobre Patrimonio Histórico Arquitectónico [1], incluye en el artículo 6 las categorías de conjunto y centro histórico para la protección del patrimonio correspondiente a grupos o asentamientos. No obstante, en la práctica lograr la declaratoria de un centro histórico mediante la Ley 7555 es un proceso complejo, porque implica hacerlo mediante un decreto que emite la Asamblea Legislativa. Esto ha llevado a que los centros históricos se protejan mediante acuerdo municipal, tal es el caso del Centro Histórico de San José, que fue declarado como perímetro urbano, y su zona urbana circundante como ensanches, en el año 2013 por parte de la Municipalidad de San José [2].

En el campo de la protección de los grupos con valor patrimonial, además de conocer los procedimientos para lograr su protección, es importante comprender la forma en que se delimitan, lo que implica conocer qué se entiende por centro histórico. Sobre este concepto, es en la Carta del Restauro, del año 1972, donde se encuentra una primera definición que se refiere a asentamientos humanos que, aunque se hayan transformado en el tiempo "... tengan particular valor de testimonio histórico, arquitectónico o urbanístico."[3] Otras cartas han brindado definiciones de centro histórico, no obstante, el concepto más reciente, y que engloba lo mencionado en las líneas anteriores, es el de paisaje urbano histórico, definido por la Conferencia General de la UNESCO en 2011 así: "Se entiende por paisaje urbano histórico la zona urbana resultante de una estratificación histórica de valores y atributos culturales y naturales, lo que trasciende la noción de "conjunto" o "centro histórico" para abarcar el contexto urbano general y su entorno geográfico." [4] El paisaje urbano histórico se presenta como una concepción integral del patrimonio, que protege tanto los elementos tangibles como intangibles, y que busca mejorar la calidad de vida de sus habitantes. Esta definición se asemeja a la idea de palimpsesto, concepto que el historiador suizo André Corboz define en relación a como el territorio se estructura en capas que responden a distintos momentos históricos [5, p. 34].

Dado que el concepto de centro histórico se mantiene vigente en la normativa de protección al patrimonio, surge la interrogante: la aceptación generalizada del concepto de centro histórico ¿lo convierte en adecuado para cualquier contexto? ¿qué sucede con las ciudades latinoamericanas y su particular desarrollo? La arquitecta argentina Marina Waisman reflexiona sobre las particularidades de la arquitectura y las ciudades de Latinoamérica en su libro El interior de la historia. Historiografía arquitectónica para uso de latinoamericanos [6]. Específicamente sobre los centros históricos, Waisman señala que, ante la existencia de una imagen idílica de una América Latina como una gran unidad cultural, se ha caído en el error de estudiar ciertos casos representativos como si constituyeran la totalidad.

Waisman indica que en muchos países de América Latina hay centros o áreas urbanas que no han consolidado una estructura que responda a la visión tradicional de centro histórico, sin embargo, tienen valor patrimonial. Waisman plantea un concepto clave para entender estas particularidades de las ciudades latinoamericanas: las discontinuidades históricas. Las discontinuidades reflejan la construcción de la ciudad en el tiempo, caracterizada por "...rupturas, como interrupciones, como desgarramientos de tejidos apenas esbozados." [6, p. 51] Ante las reflexiones de Waisman, y ante la recomendación de la UNESCO sobre la visión integral del patrimonio que encierra el concepto de paisaje urbano histórico, se evidencia la necesidad de construir herramientas que respondan a las particularidades de las ciudades latinoamericanas. 
El objetivo del análisis urbano es estudiar de forma sistemática el tejido de la ciudad. Como lo explica el arquitecto español Alfonso del Pozo y Barajas [7], el análisis urbano permite comprender los procesos de formación, cambio y permanencia en las ciudades. Pozo explica que los primeros estudios urbanos surgieron en la segunda mitad del siglo XIX, como consecuencia del pensamiento higienista, fueron el precedente de las teorías urbanísticas contemporáneas [8]. En el siglo XX, un referente importante es el arquitecto italiano Saverio Muratori y su libro Studi per una operante storia urbana di Venezia [9], publicado en el año 1960, y los estudios que posteriormente realizó, donde sistematiza y desarrolla un procedimiento específico a la solución del problema sobre cómo analizar un espacio urbano. Muratori elabora planos de los distintos momentos históricos de la ciudad de Venezia, los organiza en orden cronológico e incluye tanto la arquitectura monumental como la arquitectura menor.

El arquitecto Gianfranco Caniggia fue el heredero de Saverio Muratori. Caniggia retoma los planteamientos de Muratori sobre la lectura histórico-tipológica del ambiente. Caniggia y Gian Luigi Maffei [10] proponen un análisis que incluye el estudio de la conformación urbana y el estudio de los tipos arquitectónicos. Los aspectos que identifican con el análisis son los siguientes:

- Estructura del territorio: el territorio tiene su propia estructura natural, que incluye el relieve, la hidrografía, el clima, el régimen de lluvias, el suelo, entre otros, que determinan cada lugar.

- Trayecto matriz: es la vía de unión de un edificio con los demás, pero un trayecto también puede servir para ir de un lugar a otro sin ser utilizado para el acceso a los edificios.

- Nodo: es la unión de dos trayectos o la bifurcación de dos trayectos.

- Trayectos de implantación de edificación: se realiza en previsión de la construcción en sus márgenes.

- Trayecto de unión: une dos trayectos de implantación. Lleva a la aparición de la manzana como módulo visible.

- Trayecto de reestructuración: es un trayecto que se superpone a un tejido de edificación anterior.

- Tejido urbano: son las relaciones entre un conjunto de edificios.

- Parcela: es el módulo del tejido urbano.

- Unidades de edificación: incluyen los edificios especializados, que son los más grandes y complejos como las iglesias. También se identifica la edificación de base, que corresponde a las viviendas.

El análisis urbano desarrollado por Caniggia y Maffei permite leer la ciudad como un palimpsesto, por tanto, permite identificar las distintas capas que conforman un conjunto con valor patrimonial. Esta visión de la ciudad corresponde a una lectura desde el concepto de transculturación. La transculturación, a partir de la interpretación del autor cubano Fernando Ortiz, se entiende como "...un proceso en el cual siempre se da algo a cambio de lo que se recibe... Un proceso en el cual emerge una nueva realidad, compuesta y compleja; una realidad que no es una aglomeración mecánica de caracteres, ni siquiera un mosaico, sino un fenómeno nuevo, original e independiente". [11]

El arquitecto Felipe Hernández [12] traslada el concepto de transculturación al estudio de las ciudades latinoamericanas, sus espacios y su arquitectura. Señala que para el estudio de la arquitectura latinoamericana, usualmente se seleccionan obras de arquitectos reconocidos, que se consideran representativos, y cuya obra se ajusta a los parámetros de la arquitectura 
moderna en Europa y América. Esto ofrece una visión parcial de las heterogéneas prácticas arquitectónicas que se desarrollan en América Latina. De esta forma, la herramienta de análisis urbano propuesta, al incluir los edificios especializados y la edificación de base, permite leer los distintos lenguajes que conforman la arquitectura de la ciudad.

EI SIG es la herramienta que permite procesar información sobre conjuntos. La arquitecta Victoria Domínguez [13] explica que el primer SIG se desarrolla en el Departamento de Agricultura de Canadá en 1964, con el objetivo de estudiar el territorio rural, posteriormente, en la Universidad de Harvard, el arquitecto H. Fischer funda en 1966 el Harvard Laboratory for Computer Graphics and Spatial Analysis, lo que permitió agregar datos ecológicos, sociológicos, demográficos y espaciales. EI SIG también es considerado una herramienta adecuada tanto para el registro de las transformaciones urbanas como para el desarrollo de una estrategia de conservación, por ejemplo su aplicación en la conservación programada [14].

\section{Materiales y métodos}

Las fuentes para el desarrollo del análisis incluyen:

- Bibliografía sobre el desarrollo urbano-arquitectónico de San José, tanto libros como trabajos finales de graduación desarrollados, principalmente, en la Escuela de Arquitectura de la Universidad de Costa Rica.

- Mapas y planos de la ciudad de San José desde el siglo XIX hasta la actualidad, disponibles en el Archivo Nacional de Costa Rica.

- Fotografías aéreas de la ciudad de San José, disponibles en el Instituto Geográfico Nacional.

- Fotografías de la ciudad de San José, desde el siglo XIX hasta la actualidad, publicadas en los grupos de la red social Facebook «Fotografías Antiguas de Costa Rica», «Custodios del Patrimonio de Costa Rica», «Protejamos nuestro patrimonio arquitectónico Costa Rica» y «Salvemos el Variedades».

- Álbumes fotográficos de finales del siglo XIX e inicios del siglo XX de los fotógrafos Otto Siemon, Fernando Zamora y Manuel Gómez Miralles.

- Aplicación de una encuesta a los usuarios de la ciudad.

Las fuentes anteriores son las que permiten desarrollar el análisis urbano tomando como referencia la propuesta de Caniggia y Maffei, por lo que son el insumo para diseñar la estructura del SIG, que para efectos de esta investigación se trabajó en la plataforma QGIS, que es un software libre.

Niccolò Rapetti explica que existen tres fases en el proceso de diseño de un SIG: el diseño conceptual, el diseño lógico y el diseño físico [15]. El diseño conceptual muestra los intereses de la investigación, consiste en un esquema conceptual que describe la organización de los datos en un nivel general. El diseño lógico transforma el esquema conceptual en una representación de datos, que en este caso corresponde a las capas en las que se organiza la información. La tercera fase es el diseño físico, que corresponde a los parámetros en los que se organiza el análisis, en este caso, la definición de los atributos para cada una de las capas definidas en la fase anterior.

Las primeras capas de la cartografía se desprenden del análisis histórico. Las otras capas se construyen con la información de las declaratorias patrimoniales, para así ubicar los bienes inmuebles que cuentan con protección y que corresponden a un patrimonio oficial. A partir del análisis de los imaginarios urbanos se construye la capa que corresponde al patrimonio 
subalterno, que es el identificado por la gente pero que no cuenta con declaratoria oficial. Otras capas se construyen con información sobre las distintas celebraciones y actividades culturales que se desarrollan en el espacio público. De esta forma, el SIG propuesto se compone de las siguientes capas [16, pp. 778-779]:

1. Información Histórica $(1 . \mathrm{H})$ : se organiza en forma cronológica, desde 1738 hasta la actualidad. Incluye el registro de los cambios en la ciudad, por ejemplo, desarrollo de los cuadrantes, construcción de nuevas avenidas, construcción de edificios estatales, desarrollo de nuevos barrios, entre otros aspectos.

2. Patrimonio Oficial (2.PO): son los bienes inmuebles protegidos por la Ley 7555.

3. Patrimonio Subalterno (3.PS): es el patrimonio identificado por la gente, se refiere a los imaginarios urbanos. Es resultado de la encuesta en la que los ciudadanos indicaron los edificios, espacios y conjuntos que son representativos según su opinión.

4. Elementos Singulares (4.ES): son elementos de la ciudad que tienen valor por su representatividad y unicidad. Por ejemplo, los recorridos de los ríos, el recorrido que realizaba el tranvía y los monumentos.

5. Celebraciones Oficiales (5.CO): se refiere a celebraciones periódicas que son organizadas por entes oficiales, ya sean celebraciones nacionales como el paso de la Antorcha de la Independencia, o celebraciones organizadas por la Municipalidad de San José como el Festival de la Luz o el Carnaval en diciembre.

6. Celebraciones Religiosas (6.CR): son las celebraciones organizadas por la iglesia católica, se registra el recorrido de las celebraciones que implican el uso del espacio público, por ejemplo, la Semana Santa y la procesión del Dulce Nombre de Jesús. También se incluyen eventos que son recordados por los ciudadanos como los congresos eucarísticos.

7. Iniciativas Ciudadanas (7.IC): son actividades organizadas por la ciudadanía, por ejemplo, festivales en barrios y el Art City Tour.

8. Resultados (8.R): esta capa contiene los resultados del cruce de las capas anteriores, por ejemplo, los trayectos matrices, los trayectos de unión, edificación especializada, edificación de base, nodos y tejido urbano.

9. Fotografías Antiguas (9.FA): en esta capa se incluyen fotografías antiguas de los edificios y espacios urbanos. También se incluyen fotografías aéreas de la ciudad.

10. Fotografías Recientes (10.FR): se incluyen fotografías recientes de los edificios y espacios urbanos analizados. También incluye fotografías aéreas de la ciudad.

11. Decretos (11.D): en esta capa se adjuntan los decretos de los edificios y otros bienes que tienen declaratoria de patrimonio histórico arquitectónico.

12. Planos (12.P): capa destinada a organizar los planos sobre los edificios y sobre la ciudad. Incluye tanto material actual como material antiguo.

13. Cartografía Base (13.CB): son las capas de los cinco distritos analizados, estas capas fueron facilitadas por el Departamento de Información Catastral y Geográfica de la Municipalidad de San José. También incluye capas de ríos, distritos y provincias que fueron tomadas del Atlas Digital de Costa Rica.

Los atributos de las capas son: nombre, año, lenguaje, arquitecto, materiales, sistema constructivo, actualidad (se conserva, remodelado, reconstruido, demolido), barrio, distrito, notas, periodo (periodo en que fue construido), fuentes (referencias documentales) y nomenclatura histórica (nombres que ha tenido anteriormente). 


\section{Resultados}

A partir del análisis urbano, se obtienen los siguientes resultados [16, pp. 413-418, 484-486]. A diferencia de otras ciudades de origen colonial, San José tuvo una fundación tardía, además, la iglesia, edificio principal de los asentamientos coloniales, fue trasladada, lo que implica que el tejido urbano se consolidó hasta finales del siglo XVIII. El trayecto matriz de la ciudad es la Avenida Central, vía que en el periodo colonial unía a San José con la capital Cartago y con los pueblos de indios. Este trayecto se consolidó cuando la ciudad se extendió hacia el oeste, hasta el Llano de La Sabana, y cuando se consolidó el tejido urbano alrededor de la iglesia principal, y la avenida pasa a llamarse Calle del Comercio. Así se define el primer trayecto de implantación de la ciudad, en el cuadrante que se desarrolla alrededor de la Catedral y el Parque Central, entre avenidas 7 y 10, calles 9 y 12, y que ha sido tomado como Centro Histórico de la ciudad. Los barrios que aparecen hacia los cuatro puntos cardinales de este perímetro también se consideran trayectos de implantación.

Como trayectos de unión se consideran las vías que unen los barrios que inician el desarrollo durante el Periodo Liberal, proceso que continúa durante el siglo XX y se proyecta a la actualidad con el desarrollo de las torres habitacionales en Mata Redonda, proyectos que, si bien son contrarios a la condición de barrio, sí son parte de los cambios en el tejido urbano.

Durante el Periodo Republicano se consolidan otros dos trayectos matrices: la actual Calle Central, Ilamada La Pólvora porque se extendía hasta el Río María Aguilar, al sur, donde estaban los depósitos de pólvora. El otro eje es la calle La Alameda, actual Avenida 10, que comunicaba con el Cementerio y que posteriormente fue arborizada. En el Periodo Liberal se consolida el Paseo de Las Damas, trayecto que por su importancia en la época al comunicar con la Estación del Ferrocarril al Atlántico, es considerado un trayecto matriz.

Los dos trayectos principales (Avenida Central y Calle Central), definen en su cruce un nodo, que se enfatiza con la construcción de la línea del tranvía, que precisamente tiene por ejes principales estas dos vías. En este nodo se ubica uno de los conjuntos principales de la ciudad, conformado por la Catedral y el Parque Central. Esta condición de centralidad se ve enfatizada con la ampliación de la Avenida Segunda en la segunda mitad del siglo XX, que le da a esta avenida la condición de trayecto de unión entre dos conjuntos de la ciudad: el conjunto hospitalario al oeste y el conjunto institucional al este.

La línea férrea presenta una dualidad que se debe a que une y divide a la vez. Une dos elementos que son hitos en la ciudad y símbolo de progreso: la Estación del Ferrocarril al Pacífico y la Estación del Ferrocarril al Atlántico, por lo que se considera un trayecto de unión. Pero la línea férrea también es un elemento que simboliza la segregación social en la ciudad: al sur de la línea se desarrollaron los barrios de obreros y artesanos, que durante años lucharon por obtener los servicios básicos como agua y electricidad.

Las viviendas de los barrios son consideradas como edificación de base con características diversas según su ubicación. Así al norte se encuentran viviendas de madera y con un lenguaje arquitectónico definido como victoriano. Al sur, aunque también se utiliza la madera, son viviendas con una escala y lenguaje modestos. La edificación de base se diversifica con el desarrollo de barrios como Barrio México donde se encuentran viviendas en lenguaje art decó, o Barrio Pitahaya y Barrio Escalante, que tienen importantes ejemplos de viviendas con lenguaje moderno.

La edificación especializada se identifica hasta mediados del siglo XX con los edificios estatales. Inicialmente era arquitectura en lenguaje neoclásico, luego se introducen lenguajes como el neocolonial y el art decó. Después de 1950, con la llegada del movimiento moderno, el panorama urbano de San José cambia notoriamente. A partir de 1950 se construyen los 
primeros edificios en altura, primero dedicados a instituciones estatales, lo que también fue una forma de mostrar la consolidación del Estado luego de la Guerra Civil de 1948. A partir de 1970 aparecen edificios en altura con nuevos usos, por ejemplo, centros comerciales y hoteles. A partir del año 2000 inicia la construcción de torres de apartamentos, que se concentran principalmente en Mata Redonda, en los alrededores del Parque Metropolitano La Sabana.

La Avenida Central y la Avenida Segunda son las dos vías más importantes para quienes a diario transitan por San José. La Avenida Central es un trayecto matriz que adquirió nuevo significado en la década de 1990 con la peatonalización. La observación realizada confirmó la importante actividad que tiene esta vía, no sólo por ser comercial, sino por la existencia de zonas donde la gente puede compartir momentos de ocio. Especialmente importante es el punto de unión entre Avenida Central y Plaza de la Cultura.

La Avenida Segunda presenta una característica particular, ya que, por su ensanchamiento en la segunda mitad del siglo XX, se volvió un espacio ideal para actividades que impliquen gran concentración de personas. Por este motivo, los recuerdos de las visitas de personas como el presidente estadounidense John F. Kennedy o el Papa Juan Pablo II, se asocian a esta vía capitalina. También en la Avenida Segunda se concentran actividades como las fiestas de fin de año (Carnaval, Tope, Festival de la Luz) y fiestas cívicas como la celebración del 15 de setiembre, entre otras. Por su relación con la Catedral, también es una vía importante para las actividades religiosas, por ejemplo, las procesiones de Semana Santa.

El conjunto del Parque Central y la Catedral, y la Plaza de la Cultura, son parte fundamental del imaginario josefino. Al consultar sobre los recorridos por la ciudad, éstos son puntos de referencia muy mencionados. Si bien en ambos casos hay usuarios de todas las edades, el Parque Central-Catedral es más mencionado por personas de mayor edad, el segmento joven de la población se muestra más identificado con la Plaza de la Cultura.

La Avenida 4, que se integra al Parque Central, es otro de los recorridos más significativos para los ciudadanos. Dos son los aspectos que le han dado un nuevo significado a esta vía de la ciudad: la peatonalización y la presencia de población nicaragüense en el sector oeste del bulevar, en la zona donde se une a la iglesia y al parque de La Merced. Los nicaragüenses le dan una dinámica particular a esta zona, con la venta de productos tradicionales de su país.

Otro espacio que ha adquirido un nuevo significado es el Templo de la Música (quiosco del Parque Morazán). Este quiosco, que en su momento fue utilizado durante actividades religiosas como el Primer Congreso Eucarístico, también fue sede de las fiestas de fin de año, actualmente es utilizado por jóvenes artistas urbanos. El sector del Paseo de Las Damas es la sede de actividades culturales como el Transitarte o el Art City Tour. La actividad cultural en el Paseo de Las Damas forma parte de una serie de cambios que se han dado en el sector norte de la ciudad, en el distrito Carmen. Por ejemplo, Barrio Escalante ahora se caracteriza por la actividad gastronómica (Paseo Gastronómico).

El distrito de Mata Redonda, que como se mencionó en el análisis urbano, actualmente se caracteriza por el desarrollo inmobiliario con torres de apartamentos, pero sin mayor aporte a la calidad del espacio urbano, tiene el Parque de La Sabana, un importante punto de encuentro para actividades de ocio y deportivas. Tanto parque como Estadio Nacional y el Paseo Colón, vía que comunica La Sabana con el centro de la ciudad, tienen un espacio en la memoria de los ciudadanos, que asocian a este espacio acontecimientos como los congresos eucarísticos y la visita de Juan Pablo II y el presidente Kennedy.

Se identificaron nueve trayectos y doce conjuntos, que se muestran en la figura 1, que incluyen el patrimonio oficial y el patrimonio cotidiano, además reflejan el crecimiento de la ciudad y su condición de palimpsesto. Los trayectos son los siguientes: Avenida Central-Paseo Colón, 
Calle Central, Avenida 10, Paseo de Las Damas, Avenida Segunda, Paseo de Los Estudiantes, Boulevard Avenida 4, Línea Férrea y Ríos Torres-María Aguilar. Los conjuntos identificados son: tejido urbano del siglo XIX, centro originario, Parque Central-Catedral Metropolitana, Plaza de la Cultura-Teatro Nacional, Paseo de Las Damas, la Caja- La Soledad, Hospital-La Merced, Cementerios, Pacífico-Hospital Carit, Plaza González Víquez, Conjunto Judicial y La Sabana.



Figura 1: Trayectos y conjuntos con valor patrimonial en la ciudad de San José. Elaboración propia sobre cartografía base proporcionada por la Municipalidad de San José y el Atlas Digital de Costa Rica.

\section{Conclusiones}

A partir del concepto de transculturación se comprende la diversidad arquitectónica y urbana de la ciudad de San José, un palimpsesto en el que convive el lenguaje moderno con edificios del siglo XIX y una arquitectura menor generalmente ignorada. Al aplicar el concepto de transculturación surge la pregunta: ¿son considerados con valor patrimonial la arquitectura y los espacios urbanos producto de un proceso de transculturación, o en la identificación de bienes patrimoniales se considera solamente una parte de esta realidad?

Ni la construcción oficial ni la construcción cotidiana del patrimonio son totalmente incluyentes con las manifestaciones patrimoniales, como lo explica Beatriz Sarlo [17], la memoria es anacrónica, se recuerda lo que se desea recordar, o lo que interesa a los grupos con poder que sea recordado. Sin embargo, a pesar de las exclusiones a las que puede llevar el anacronismo de la memoria, se puede afirmar que San José es imaginado mediante imaginarios posmodernos, concepto acotado por Daniel Hiernaux[18] que se refiere a la convivencia de pasado y presente, situación que es consecuencia de la condición de palimpsesto urbano. 
Caniggia y Maffei consideran que la idea de centro histórico deja fuera las expansiones de la ciudad durante el siglo XX, considerando estas zonas como "no históricas" [10, p. 182]. Aunque esta es una observación para las ciudades europeas, que después del derribo de las murallas a finales del siglo XIX expandieron sus límites, al trasladar la reflexión a San José, se comprende que la necesidad de contar con una herramienta de análisis que permita vislumbrar la discontinuidad propia del proceso de formación de la ciudad.

Caniggia y Maffei proponen que en la ciudad hay ambientes consolidados y ambientes en vías de consolidación. Esto introduce otra variable para tener en cuenta al momento de sugerir estrategias para la conservación del patrimonio, porque la ciudad no es estática, está en constante cambio. En San José se considera que los ambientes consolidados se encuentran en el núcleo urbano elemental, donde se ha desplazado a los habitantes para dar lugar a edificios comerciales e institucionales. Los barrios se consideran ambientes en vías de consolidación, porque constantemente experimentan cambios, lamentablemente muchos no son favorables como es el desplazamiento de la población por problemas sociales, como sucede en los barrios del sur, o por la presencia de comercio como sucede en otros sectores de la ciudad. Otro ejemplo de ambiente en vía de consolidación se encuentra en los sectores donde se están construyendo las torres de apartamentos, que por su escala y negación del espacio urbano, implican un profundo cambio en los barrios donde se ubican.

El Análisis Urbano realizado debe ser ampliado a futuro, específicamente para desarrollar el estudio de las tipologías arquitectónicas, tema que en esta investigación no se realizó por no formar parte de su alcance. También es necesario extender el análisis a los barrios, que son considerados como ambientes en vías de consolidación y conformados por arquitectura de base.

\section{Agradecimientos}

Este texto es producto de la tesis doctoral "La construcción social del patrimonio urbano y arquitectónico en la ciudad de San José, Costa Rica. Estrategias para su conocimiento", presentada en la Escuela Técnica Superior de Arquitectura de la Universidad de Sevilla el 24 de junio de 2019, y cuya realización fue posible gracias a la beca de estudios otorgada por el Instituto Tecnológico de Costa Rica. Se agradece la guía recibida para la elaboración de la tesis doctoral por parte de los profesores Dr. Francisco González de Canales Ruiz, de la Universidad de Sevilla, y del Prof. Arch. Marco Pretelli, de la Universidad de Bologna.

\section{Referencias}

[1] Costa Rica, «Ley № 7555 de Patrimonio Histórico Arquitectónico», 1995. http://icomoscr.org/content/index. php?option=com_content\&view=article\&id=60\&ltemid=69.

[2] Municipalidad de San José, «Acta de la Sesión Ordinaria celebrada por la Corporación Municipal del Cantón Central de San José». San José, Costa Rica, 2013.

[3] Ministerio de Instrucción Pública de Italia, «Carta del Restauro», Roma, 1972.

[4] UNESCO, Ed., «Recomendación sobre el paisaje urbano histórico, con inclusión de un glosario de definiciones», en Actas de la Conferencia General. 36a reunión. Volumen 1, resoluciones, París: UNESCO, 2011, pp. 60-65.

[5] A. Corboz, «El territorio como palimpsesto», en Lo urbano en 20 autores contemporáneos, Á. Martín Ramos, Ed. Barcelona: Edicions de la Universitat Politècnica de Catalunya, 2004, pp. 25-34.

[6] M. Waisman, El interior de la historia: historiografía arquitectónica para uso de latinoamericanos. Bogotá: Escala, 1990.

[7] A. del Pozo y Barajas, Ed., Análisis urbano. Textos: Gianfranco Caniggia, Carlo Aymonino, Massimo Scolari. Sevilla: Universidad de Sevilla, Instituto Universitario de Ciencias de la Construcción, 1997. 
[8] A. del Pozo y Barajas, Arrabales de Sevilla, morfogénesis y transformación. El Arrabal de Los Humeros. Sevilla: Junta de Andalucía, Consejería de Obras Públicas y Transportes. Universidad de Sevilla, Secretariado de Publicaciones, 1996.

[9] S. Muratori, Studi per una operante storia urbana di Venezia. Roma: Istituto Poligrafico dello Stato, 1960.

[10] G. Caniggia y G. L. Maffei, Tipología de la edificación. Estructura del espacio antrópico. Madrid: Celeste, 1995.

[11] F. Ortiz, Contrapunteo cubano del tabaco y el azúcar. Barcelona: Ariel, 1973.

[12] F. Hernández, M. Millington, y I. Borden, Eds., Transculturation Cities, Spaces and Architectures in Latin America. Amsterdam: Rodopi, 2005.

[13] V. Domínguez Ruiz, «Aplicación de los Sistemas de Información Geográfica (SIG) al proyecto de rehabilitación arquitectónica y urbana. », Universidad de Sevilla, Doctorado en Arquitectura, 2015.

[14] L. Baratin, S. Bertozzi, y E. Moretti, «Tecnologia GIS per la manutenzione programmata dei beni culturali», en Proceedings of the International Conference Preventive and Planned Conservation. ICT per il miglioramento del processo conservativo., 2014, vol. 5, pp. 73-84.

[15] N. Rapetti, «II GIS per la gestione dei dati alla scala territoriale», en Building Information Modeling, Geographic Information System, Augmented Reality per il facility management, A. Osello, Ed. Palermo: Dario Flaccovio Editore, 2015, pp. 233-240.

[16] R. E. Malavassi Aguilar, «La construcción social del patrimonio urbano y arquitectónico en la ciudad de San José, Costa Rica. Estrategias para su conocimiento.», Tesis Doctorado en Arquitectura, Universidad de Sevilla, 2019.

[17] B. Sarlo, Tiempo pasado. Cultura de la memoria y giro subjetivo. Una discusión. Buenos Aires: Siglo XXI Editores, 2005.

[18] D. Hiernaux, «De la espacialidad, el lugar y los imaginarios urbanos: a modo de introducción», en Lugares e imaginarios en la metrópolis, A. Lindón, M. Á. Aguilar, y D. Hiernaux, Eds. Barcelona; México: Anthropos Editorial; Universidad Autónoma Metropolitana, 2006, pp. 27-41. 